\title{
Rehabilitation in patients with radically treated respiratory cancer: A randomised controlled trial comparing two training modalities
}

\author{
Bihiyga Salhi $^{\mathrm{a}, *}$, Christel Haenebalcke ${ }^{\mathrm{b}}$, Silvia Perez-Bogerd ${ }^{\mathrm{c}}$, Mai D. Nguyen ${ }^{\mathrm{d}}$, \\ Vincent Ninane $^{c}$, Thomas L.A. Malfait ${ }^{a}$, Karim Y. Vermaelen ${ }^{a}$, Veerle F. Surmont ${ }^{a}$, \\ Georges Van Maele ${ }^{\mathrm{e}}$, Roos Colman ${ }^{\mathrm{e}}$, Eric Derom ${ }^{\mathrm{a}}$, Jan P. van Meerbeeck ${ }^{\mathrm{a}, \mathrm{f}}$ \\ a Department of Respiratory Medicine, Ghent University Hospital, Belgium \\ ${ }^{\mathrm{b}}$ Department of Respiratory Medicine, AZ Sint Jan, Bruges, Belgium \\ ${ }^{\mathrm{c}}$ Department of Respiratory Medicine, CHU St Pierre, Brussels, Belgium \\ ${ }^{\mathrm{d}}$ Department of Respiratory Medicine, CHU Sart Tilman, Liège, Belgium \\ e Biostatistical Unit, Faculty of Medicine, Ghent University, Belgium \\ ${ }^{\mathrm{f}}$ Thoracic Oncology, Antwerp University Hospital, Belgium
}

\section{A R T I C L E I N F O}

Article history:

Received 8 February 2015

Received in revised form 12 May 2015

Accepted 14 May 2015

\section{Keywords:}

Lung cancer

Radical treatment

Pulmonary rehabilitation

Resistance training

Whole body vibration

Exercise

\begin{abstract}
A B S T R A C T
Introduction: The evidence on the effectiveness of rehabilitation in lung cancer patients is limited. Whole body vibration (WBV) has been proposed as an alternative to conventional resistance training (CRT). Methods: We investigated the effect of radical treatment (RT) and of two rehabilitation programmes in lung cancer patients. The primary endpoint was a change in 6-min walking distance (6MWD) after rehabilitation. Patients were randomised after RT to either CRT, WBVT or standard follow-up (CON). Patients were evaluated before, after RT and after 12 weeks of intervention.

Results: Of 121 included patients, 70 were randomised to either CON (24), CRT (24) or WBVT (22). After RT, 6MWD decreased with a mean of $38 \mathrm{~m}(95 \% \mathrm{CI} 22-54)$ and increased with a mean of $95 \mathrm{~m}(95 \% \mathrm{CI}$ $58-132)$ in CRT $(p<0.0001), 37 \mathrm{~m}(95 \% \mathrm{CI}-1-76)$ in WBVT $(p=0.06)$ and $1 \mathrm{~m}(95 \% \mathrm{CI}-34-36)$ in CON $(p=0.95)$, respectively. Surgical treatment, magnitude of decrease in 6MWD by RT and allocation to either CRT or WBVT were prognostic for reaching the minimally clinically important difference of $54 \mathrm{~m}$ increase in 6MWD after intervention.

Conclusions: RT of lung cancer significantly impairs patients' exercise capacity. CRT significantly improves and restores functional exercise capacity, whereas WBVT does not fully substitute for CRT.
\end{abstract}

(c) 2015 Elsevier Ireland Ltd. All rights reserved.

\section{Introduction}

A minority of patients with lung cancer receives a treatment with curative intent, consisting of either radical surgery or definitive radiotherapy, administered either as single modality or combined with platinum-based chemotherapy [11,33,34].

These treatments lead to a decrease in QoL, physical activity and enhance their morbidity [10]. Cancer-related fatigue (CRF), which is frequently reported by cancer patients, is defined as an unusual and persistent sense of tiredness, affecting both physical and mental capacity and is unrelieved by rest [35]. The underlying mechanisms are biological (anaemia, pro-inflammatory

\footnotetext{
* Corresponding author at: Department of Respiratory Medicine, Ghent University Hospital, De Pintelaan 185, 7K12IE, B-9000 Ghent, Belgium. Tel.: +32 9332 5536; fax: +32 93322341 .

E-mail address: Bihiyga.Salhi@UGent.be (B. Salhi).
}

cytokines, nutritional and fluid imbalances, muscle wasting), functional (reduced aerobic capacity and decreased activity of daily living) and psycho-behavioural (sleep disorders, anxiety, depression, reduced self-efficacy, sleep disorders, distress and difficulty coping). This may lead to a further muscle deconditioning and disuse atrophy [35], which in turn may aggravate the feeling of fatigue [1].

Oncological rehabilitation has most been extensively studied in breast cancer patients [28]. The beneficial effects of rehabilitation in lung cancer patients, were currently limited to a few randomised trials. These trials showed that patients with lung cancer can improve their exercise capacity, muscle strength and QoL, however the results were not consistent $[2,9,29]$.

Whole body vibration training (WBVT) has been proposed as an alternative training modality for resistance training on multigym equipment. WBVT generates vertical sinusoidal vibrations and elicits in short periods reflectory neuromuscular training without much effort [26]. It is assumed that these vibrations evoke muscle 
contractions via a tonic vibration reflex [32]. In elderly subjects, WBVT improved both aerobic fitness and muscle strength [4].

The present multi-centre trial, acronamed "REINFORCE" (Randomized Exercise trainINg FOr patients with Radically treated respiratory CancEr), was designed to assess the potential beneficial effect of rehabilitation in lung cancer patients. More specifically, we wanted to address the following questions: (1) does lung cancer therapy affect exercise capacity, muscle strength and QoL; (2) does a 12 -week rehabilitation programme improve $6 \mathrm{MWD}$ (the primary outcome), maximal exercise capacity, muscle strength and QoL; and (3) are both training methods, WBVT and conventional resistance training (CRT), equally effective in improving 6MWD and other outcome variables?"

\section{Materials and methods}

Sequential patients with stages I-III lung cancer or mesothelioma, candidate for a treatment with curative intent, were solicited by their attending physician of four departments of Respiratory Medicine to participate in the present study. Radical treatment was defined as either radical resection with or without a perioperative platinum-based chemo-(radio) therapy, or definitive thoracic radiotherapy with or without concurrent or sequential platinum-based induction chemotherapy. Patients were between 18 and 80 years and had a baseline haemoglobin level of at least $8 \mathrm{~g} / \mathrm{dl}$. Patients with severe cachexia (a decrease of at least $35 \%$ premorbid weight), co-morbidities interfering with exercise training and contra-indications for WBVT, such as a pacemaker, joint prostheses or recently introduced osteosynthetic material and osteoporotic fractures were excluded. All patients provided written informed consent at inclusion. The study was approved by the Ethics Committee of each participating hospital.

This open multi-centre trial consisted of a prospective observational part I, describing the effect of radical treatment and a randomised part II, analysing the effect of the intervention in those patients who were radically treated. In part I, patients were evaluated before (M1) and after (M2) radical treatment. M2 was assessed within 8 weeks of resection or within 2 weeks after the end of the non-surgical treatment. Patients proceeded only to part II, if their treatment was considered radical and if their post-treatment quadriceps force (QF) was either equal or less than $70 \%$ of the predicted normal value or showed a decrease of at least $10 \%$ from the baseline value [8]. The randomisation procedure was conducted directly after the M2 evaluation.

Patient randomisation was conducted by a blinded, web-based platform using a minimisation technique with surgery, COPD and centre as stratification variables and with random allocation to either a control group (CON), a CRT-group and a WBVT-group. Patients allocated to CON were discouraged to improve their exercise tolerance with professional help. Patients allocated to either CRT and WBVT received 20 min of aerobic training on the bicycle and treadmill at $70 \%$ of the respective maximal workload (Wmax) and speed, observed at M2. Thereafter, CRT-group received resistance training on multigym equipment starting with three sets of eight repetitions for each exercise at $50 \%$ one-repetitionmaximum (1RM) (Appendix 1). WBVT-group performed exercises on the vibration platform (FITVIBE, Gymna, Belgium), starting with three sets of $30 \mathrm{~s}$ for each exercise at $27 \mathrm{~Hz}$. Rehabilitation started within 8 days after randomisation. Patients trained three times a week for 12 weeks, whereafter they were re-evaluated (M3). The investigator was unblinded for the intervention and its evaluation.

The Charlson comorbidity index was used to reflect comorbidities [7]. Spirometry, diffusion capacity (DL,CO) and 6MWD with continuous oxygen saturation monitoring were measured according to existing guidelines and expressed as percentage predicted $[5,19,23,30]$. A change of at least $54 \mathrm{~m}$ in $6 \mathrm{MWD}$ was considered as the minimally clinically important difference (MCID) [24]. Maximal exercise capacity was assessed by Wmax and $\mathrm{VO}_{2}$ peak using an incremental symptom-limited cycle ergometer test and compared with normal values [18]. A change exceeding $10 \mathrm{~W}$ was considered as MCID [25]. QF was assessed using an isometric handheld dynamometer (Microfet; Biometrics, Almere, the Netherlands) attached to a knee pendicular bank. Extension peak torque was evaluated at $60^{\circ}$ of knee flexion, by performing a $5 \mathrm{~s}$ maximal isometric contraction. The best out of three attempts was retained. Health-related QoL was measured by the European Organisation for Research and Treatment of Cancer Quality of Life Cancer Questionnaire (EORTC QLQ-C30) and more specifically the item physical functioning (PF) [14]. Fatigue was assessed by the Functional Assessment of Cancer Therapy-Fatigue (FACT-F) [6,37]. Pain and dyspnoea were scored with visual analogue scales (VAS) [13].

The adherence of the trial was defined as the percentage of patients completing the intervention. It was calculated as ratio between the number of patients, who did not drop out and the total number of patients who were randomised to the active intervention. The attendance was defined as the percentage of attended sessions of the proposed 36 sessions. At each supervised training session, the study-intervention-related adverse events were recorded.

\section{Statistical analysis}

Baseline patient characteristics are expressed as medians with ranges. The effect of radical treatment was analysed in all participants completing part I (sample 1). The primary endpoint of the trial is the change in 6MWD $(\mathrm{m})$ in those patients who proceeded to part II (sample 2). The null hypothesis is that neither CRT nor WBVT would result in an increase of at least $54 \mathrm{~m}$ in 6MWD, the proposed MCID [24]. To refute this, a sample size of 57 patients (19 patients in each group) is needed ( $\alpha$ : 0.05; power: 0.80) [3]. Assuming a dropout rate of $50 \%$ of patients after part I, 114 participants had to be included in the study.

The primary endpoint was analysed by performing an intentionto-treat (ITT)-analysis on sample 2. A per protocol (PP)-analysis on patients who completed part II, defined as sample 3, was also conducted. For the ITT-analysis, missing observations at M3 were predicted by applying multiple imputations using monotone linear regression (Proc MI in SAS 9.3). Linear regression was applied on 50 imputed datasets and the results were combined using SAS Proc Mianalyse to calculate means with 95\% confidence intervals (CI) [38]. Bonferroni corrections were applied to correct for multiple pairwise comparisons.

The effect of radical treatment and the combined effect of radical treatment and intervention, both expressed as changes in exercise capacity, muscle strength and QoL, were analysed with the paired- $T$ test for differences within, and by one-way ANOVA for differences in-between groups. These results were expressed as means with $95 \% \mathrm{CI}$ (SPSS version 20 , Chicago IL). In order to analyse variables predictive for reaching the MCID in 6MWD, the allocation to either CRT or WBVT, together with relevant clinical factors, were combined in a multiple logistic regression model on sample 3. All comparisons were done with the use of a two-sided $\alpha$ level of 0.05 .

\section{Results}

\subsection{Patient population}

Between January 2009 and February 2012, 121 consecutive patients were recruited (Fig. 1). Eighty-six patients completed part 


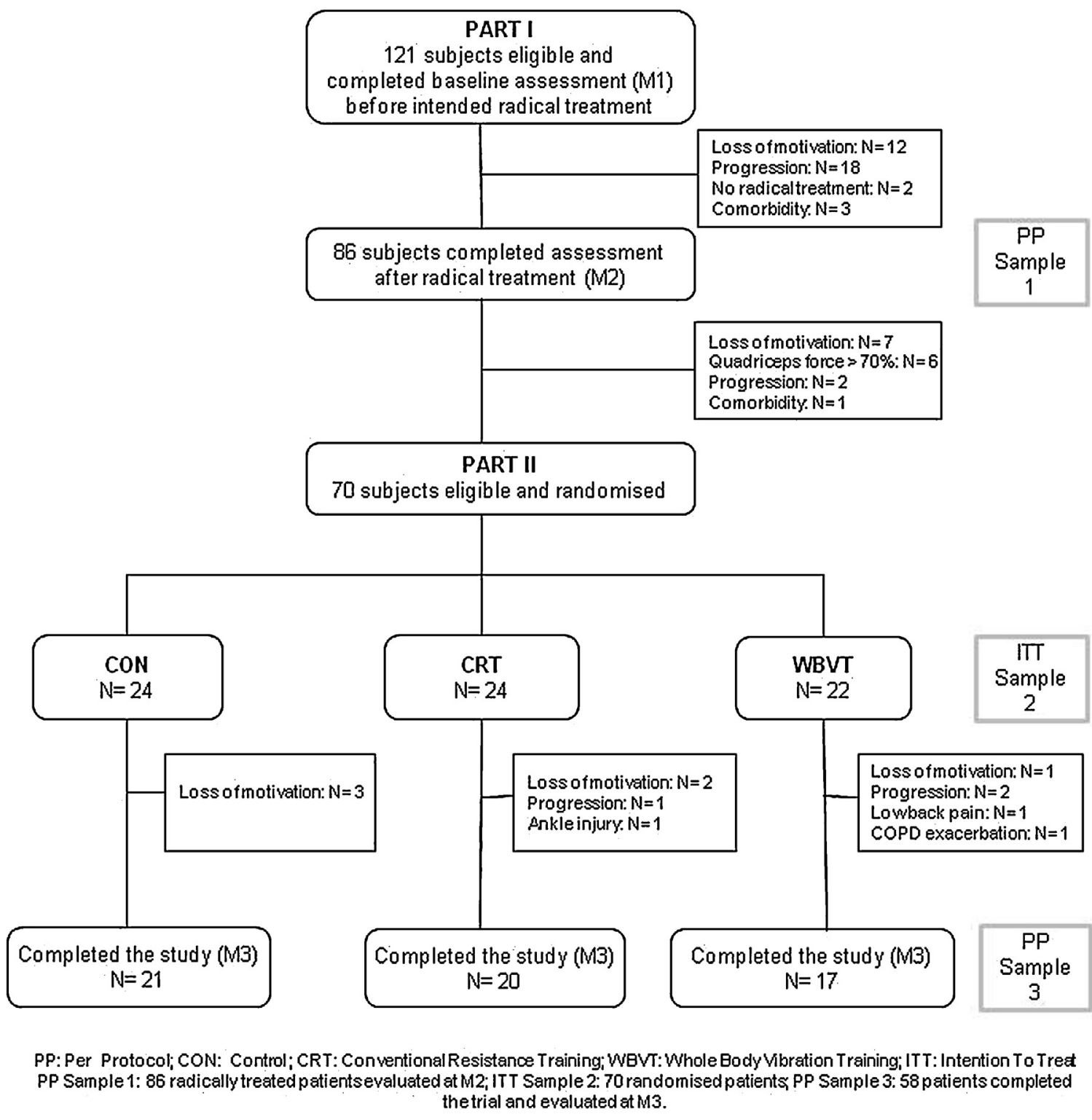

Fig. 1. CONSORT flow diagram.

I and constitute sample 1 . Before randomisation, 16 additional patients dropped out. Seventy radically treated patients were randomly assigned to either CON $(n=24)$, CRT $(n=24)$ or WBVT $(n=22)$ (sample 2). Of these patients, 21,20 and 17, respectively, completed the intervention (sample 3 ). Eighty percent of patients completed the rehabilitation programme. Twelve patients dropped out before M3 because of loss of motivation $(n=6)$, progression of disease $(n=3)$, an acute COPD exacerbation $(n=1)$, an ankle injury unrelated to the intervention $(n=1)$ and worsening of pre-existing low back pain, probably not related to the training $(n=1)$. No study-intervention-related adverse events were observed during part II.

The baseline clinical features of sample 1 and the three intervention groups were well balanced (Table 1a) with 6MWD averaging $75 \%$ of the predicted value (Table $1 \mathrm{~b})$. Significant differences in patient characteristics between the randomised patients $(N=70)$ and the non-randomised patients $(N=51)$ were not observed, except for median $\mathrm{VO}_{2}$ peak $(1.40 \mathrm{~L} / \mathrm{min}(0.8-1.8)$ vs. $1.24 \mathrm{~L} / \mathrm{min}$ (0.4-2.4) (Supplementary appendix 1).

\subsection{Effect of radical treatment $(\Delta M 2-M 1)$ in sample $1(\mathrm{~N}=86)$}

The median interval between M1 and M2 was 13 weeks (6-35). Radical treatment decreased the $\mathrm{FEV}_{1}$ with a mean of $0.4 \mathrm{~L}(95 \%$ CI $0.3-0.5)(p<0.001)$, the DL,CO expressed as a \% of the predicted value decreased with $16 \%(95 \% \mathrm{CI} 13-20)(p<0.001)$ and the 6MWD with a mean of $38 \mathrm{~m}(95 \% \mathrm{CI} 22-54)(p<0.001)$ (Table 2). Maximal exercise capacity, QF and QoL were also significantly affected.

\subsection{Effect of rehabilitation $(\Delta M 3-M 2)$ in sample $2(\mathrm{~N}=70)$}

The median interval between M2 and M3 was 13 (5-30), 14 (7-36) and 13 weeks (6-37) for CON, CRT and WBVT, respectively. Of the proposed 36 sessions, CRT-patients attended a median of $28(10-36)$ and WBVT-patients a median of 23 sessions (0-36) $(p=0.09)$.

With respect to the primary endpoint, 6MWD increased with a mean of $1 \mathrm{~m}(95 \% \mathrm{CI}-34-36 ; \mathrm{pp}=0.95)$ in CON, $95 \mathrm{~m}(95 \% \mathrm{CI}$ 58-132; $p<0.0001)$ in CRT and $37 \mathrm{~m}(95 \% \mathrm{CI}-1-76$; $\mathrm{p} p=0.06)$ in 
Table 1a

Baseline clinical characteristics of sample 1 and the three intervention groups.

\begin{tabular}{|c|c|c|c|c|}
\hline & $\begin{array}{l}\text { PART I } \\
(N=86)\end{array}$ & $\begin{array}{l}\mathrm{CON} \\
(N=24)\end{array}$ & $\begin{array}{l}\text { CRT } \\
(N=24)\end{array}$ & $\begin{array}{l}\text { WBVT } \\
(N=22)\end{array}$ \\
\hline Male $(N)$ & $64(74.4)$ & $18(75.0)$ & $18(75.0)$ & $15(68.2)$ \\
\hline Median age (years) & $63[29-84]$ & $64[51-79]$ & $63[29-76]$ & $60[38-77]$ \\
\hline Median BMI $\left(\mathrm{kg} / \mathrm{m}^{2}\right)$ & $25[16-45]$ & $26[18-35]$ & $26[17-45]$ & 23 [16-29] \\
\hline Median Charlson Comorbidity Index (points) & $3[0-11]$ & $3[1-7]$ & $3[0-10]$ & $3[0-7]$ \\
\hline $\operatorname{COPD}(N)$ & $36(41.9)$ & $9(37.5)$ & $11(45.8)$ & $9(40.9)$ \\
\hline \multicolumn{5}{|l|}{ Smoking status $(N)$} \\
\hline Never smoker & $5(5.8)$ & $1(4.2)$ & $2(8.3)$ & $2(9.1)$ \\
\hline Ex-smokers & $48(55.8)$ & $12(50)$ & $14(58.3)$ & $12(54.5)$ \\
\hline Current smoker & $33(38.4)$ & $11(45.8)$ & $8(33.3)$ & $8(36.4)$ \\
\hline Pack years $(N)$ & $40[0-100]$ & $40[0-80]$ & $40[0-100]$ & $35[0-70]$ \\
\hline \multicolumn{5}{|l|}{ Diagnosis $(N)$} \\
\hline NSCLC & $79(91.9)$ & $23(95.8)$ & $22(91.7)$ & $19(86.4)$ \\
\hline SCLC & $4(4.7)$ & $1(4.2)$ & 0 & $3(13.6)$ \\
\hline Mesothelioma & $3(3.5)$ & 0 & $2(8.3)$ & 0 \\
\hline \multicolumn{5}{|l|}{ Clinical stage $(N)$} \\
\hline NSCLC I & $44(52.2)$ & $15(62.5)$ & $10(41.6)$ & $12(54.5)$ \\
\hline NSCLC II & $16(18.6)$ & $2(8.3)$ & $7(29.2)$ & $4(18.1)$ \\
\hline NSCLC III & $17(22.1)$ & $6(25)$ & $5(20.9)$ & $3(13.6)$ \\
\hline NSCLC IV & 0 & 0 & 0 & 0 \\
\hline SCLC limited & $4(4.7)$ & $1(4.2)$ & 0 & $3(13.6)$ \\
\hline Mesothelioma I-III & $3(3.5)$ & 0 & $2(8.3)$ & 0 \\
\hline \multicolumn{5}{|l|}{ Treatment $(N)$} \\
\hline Any surgery & $45(52.4)$ & $15(62.5)$ & $10(41.6)$ & $13(59)$ \\
\hline Surgery with chemotherapy and/or radiotherapy & $21(24.4)$ & $3(12.5)$ & $8(33.4)$ & $5(22.8)$ \\
\hline Lobectomy & $50(58)$ & $15(83.5)$ & $12(66.5)$ & $14(77.7)$ \\
\hline Pneumonectomy & $16(16.6)$ & $3(16.5)$ & $6(33.5)$ & $4(22.3)$ \\
\hline Radiotherapy & $5(5.8)$ & $1(4.2)$ & $3(12.5)$ & 0 \\
\hline Radiotherapy and chemotherapy & $15(17.4)$ & $5(208)$ & $3(12.5)$ & $4(18.2)$ \\
\hline
\end{tabular}

Table 1b

Baseline assessment of sample 1 and the three intervention groups.

\begin{tabular}{|c|c|c|c|c|}
\hline & $\begin{array}{l}\text { PART I } \\
(N=86)\end{array}$ & $\begin{array}{l}\mathrm{CON} \\
(N=24)\end{array}$ & $\begin{array}{l}\text { CRT } \\
(N=24)\end{array}$ & $\begin{array}{l}\text { WBVT } \\
(N=22)\end{array}$ \\
\hline Median $\mathrm{FEV}_{1}(\mathrm{~L})$ & $2.50[0.79-4.65]$ & $2.40[1.28-4.18]$ & $2.55[0.79-4.65]$ & $2.68[1.39-3.61]$ \\
\hline Median DL,CO (\% predicted) & $72[36-154]$ & $81[43-154]$ & $70[36-107]$ & $64[42-101]$ \\
\hline Median 6MWD (m) & $510[232-671]$ & $525[232-671]$ & $490[385-625]$ & $522[375-645]$ \\
\hline Median 6MWD (\% predicted) & $75[40-100]$ & $77[40-100]$ & 73 [60-89] & $76[56-95]$ \\
\hline Median $\mathrm{VO}_{2}$ peak $(\mathrm{L} / \mathrm{min})$ & $1.4[0.3-2.5]$ & $1.35[0.79-2.48]$ & $1.39[0.69-2.4]$ & $1.34[0.84-2.23]$ \\
\hline Median Wmax (W) & $99[36-226]$ & $90[36-220]$ & $100[50-226]$ & $100[56-178]$ \\
\hline Median QF (Nm) & $96[28-227]$ & $97[28-227]$ & $90[48-186]$ & $102[50-184]$ \\
\hline Median EORTC QLQ-C 30, PF (points) & $87[13-100]$ & $93[13-100]$ & $87[20-100]$ & $87[13-100]$ \\
\hline Median FACT-F (points) & $9[0-42]$ & $7[4-42]$ & $10[3-40]$ & $11[4-29]$ \\
\hline Median VAS pain (points) & $0[0-9]$ & $1[0-9]$ & $0[0-7]$ & $1[0-9]$ \\
\hline Median VAS dyspnoea (points) & $0[0-9]$ & $1[0-9]$ & $1[0-7]$ & $3[0-9]$ \\
\hline
\end{tabular}

M1: at inclusion; sample 1 : subjects assessed at M1 and M2.

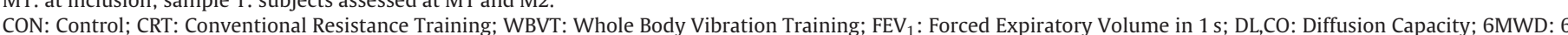

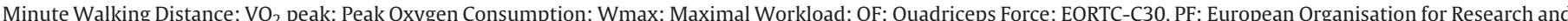

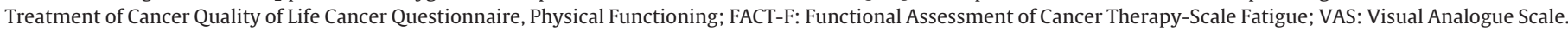

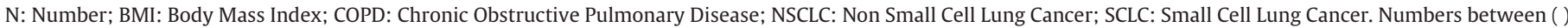
are percentages; Numbers between [ ] are ranges.

WBVT (Table 3, Fig. 2). Fifteen (75\%) of the CRT, 5(29\%) of the WBVT and 5 of the CON (24\%) patients reached the MCID for 6MWD. The mean change in 6MWD between CRT and CON was the only significant observation $(p=0.002)$. The PP-analysis showed similar results (Supplementary appendix 2).

The mean change in Wmax was significantly increased in both CRT and WBVT (15W (95\%CI 6-24); pp $=0.002$ in both groups). Seventy-four percent of CRT, $63 \%$ of WBVT and $50 \%$ of CON reached the MCID for Wmax.

QF significantly increased with a mean of $23 \mathrm{Nm}(95 \% \mathrm{CI} 10-36)$ $(p=0.0009)$ in CRT only.

For none of the groups, the score of QLQ-C30 Global and FACT-F changed significantly. However by combining the active intervention groups (CRT and WBVT) a significant increase was observed for both QLQ-C30 Global (+7 points (95\% CI 0-14), $p=0.04$ ) and
FACT-F ( -3 points $(95 \% \mathrm{CI}-5-0), p=0.04)$. No differences were observed between the active intervention groups and the control group (Table 4). The score for PF and dyspnoea improved only significantly in WBVT (PF: $p=0.04$; dyspnoea: $p=0.009$ ). The scores for pain did not significantly change after 12 weeks of intervention.

\subsection{Overall-effect ( $\triangle M 3-M 1)$ and multiple logistic regression in sample $3(\mathrm{~N}=58)$}

The 6MWD did not recover in CON-patients, the mean difference between M1 and M3 remaining $-46 \mathrm{~m}$ (95\%CI -105-13) $(p=0.12)$. The 6MWD increased with $36 \mathrm{~m}(95 \% \mathrm{CI} 1-71)$ in CRTpatients $(p=0.048)$ and $16 \mathrm{~m}(95 \% \mathrm{CI}-20-52)$ in WBVT-patients $(p=0.35)$. 
Table 2

Effect of radical treatment (sample 1).

\begin{tabular}{|c|c|c|c|c|}
\hline & $\begin{array}{l}\text { M1 } \\
\text { Mean } \\
(95 \% \mathrm{CI})\end{array}$ & $\begin{array}{l}\text { M2 } \\
\text { Mean } \\
(95 \% \mathrm{CI})\end{array}$ & $\begin{array}{l}\triangle \mathrm{M} 2-\mathrm{M} 1 \\
\text { Mean } \\
(95 \% \mathrm{CI})\end{array}$ & $P$ value $^{\mathrm{a}}$ \\
\hline Mean $\mathrm{FEV}_{1}(\mathrm{~L})$ & $2.49[2.31-2.66]$ & $2.09[1.94-2.24]$ & $-0.4[0.3-0.5]$ & $0<0.0001$ \\
\hline Mean DL,CO (\% predicted) & $75[70-80]$ & $59[54-63]$ & $-16[13-20]$ & $0<0.0001$ \\
\hline Mean 6MWD $(\mathrm{m})$ & $508[491-525]$ & $471[451-490]$ & $-38[22-54]$ & $0<0.0001$ \\
\hline Mean 6MWD (\% predicted) & $77[74-79]$ & $71[68-74]$ & $-5[3-8]$ & $0<0.0001$ \\
\hline Mean $\mathrm{VO}_{2}$ peak $(\mathrm{L} / \mathrm{min})$ & $1.39[1.31-1.48]$ & $1.16[1.07-1.25]$ & $-0.6[0.2-0.3]$ & $0<0.0001$ \\
\hline Wmax (W) & $105[96-114]$ & $85[78-93]$ & $-20[15-25]$ & $0<0.0001$ \\
\hline Mean QF (Nm) & $102[93-111]$ & $90[83-98]$ & $-11[5-18]$ & 0.001 \\
\hline Mean EORTC QLQ-C 30, PF (points) & $83[79-87]$ & $73[69-78]$ & $-9[5-14]$ & $<0.0001$ \\
\hline Mean FACT-F (points) & $11[9-13]$ & $13[11-15]$ & $2[0-4]$ & 0.02 \\
\hline Mean VAS pain (points) & $2[1,2]$ & $3[2,3]$ & $1[0-2]$ & 0.05 \\
\hline Mean VAS dyspnoea (points) & $3[2,3]$ & $3[3,4]$ & $3[3,4]$ & 0.03 \\
\hline
\end{tabular}

M1: at inclusion; M2: after radical treatment; sample 1: per protocol sample $(N=86)$, subjects assessed at M1 and M2.

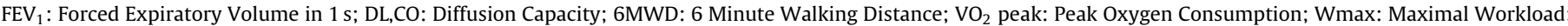

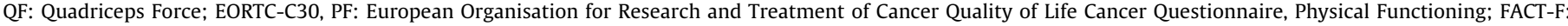
Functional Assessment of Cancer Therapy-Scale Fatigue; VAS: Visual Analogue Scale.

Numbers between [] as $95 \% \mathrm{CI}$.

a Paired- $T$ test $\Delta \mathrm{M} 2-\mathrm{M} 1$.

Table 3

Effect of the intervention in 70 randomised patients (sample 2).

\begin{tabular}{|c|c|c|c|c|c|c|c|c|c|c|c|c|}
\hline & \multicolumn{4}{|l|}{$\operatorname{CON}(N=24)$} & \multicolumn{4}{|l|}{ CRT $(N=24)$} & \multicolumn{4}{|l|}{ WBVT $(N=22)$} \\
\hline & $\begin{array}{l}\text { M2 } \\
\text { Mean } \\
(95 \% \mathrm{CI})\end{array}$ & $\begin{array}{l}\text { M3 } \\
\text { Mean } \\
(95 \% \mathrm{CI})\end{array}$ & $\begin{array}{l}\Delta \mathrm{M} 3-\mathrm{M} 2 \\
\text { Mean } \\
(95 \% \mathrm{CI})\end{array}$ & $P$ value ${ }^{\mathrm{a}}$ & $\begin{array}{l}\text { M2 } \\
\text { Mean } \\
(95 \% \mathrm{CI})\end{array}$ & $\begin{array}{l}\text { M3 } \\
\text { Mean } \\
(95 \% \mathrm{CI})\end{array}$ & $\begin{array}{l}\Delta \mathrm{M} 3-\mathrm{M} 2 \\
\text { Mean } \\
(95 \% \mathrm{CI})\end{array}$ & $P$ value $^{\mathrm{a}}$ & $\begin{array}{l}\text { M2 } \\
\text { Mean } \\
(95 \% \mathrm{Cl})\end{array}$ & $\begin{array}{l}\text { M3 } \\
\text { Mean } \\
(95 \% \mathrm{CI})\end{array}$ & $\begin{array}{l}\Delta \mathrm{M} 3-\mathrm{M} 2 \\
\text { Mean } \\
(95 \% \mathrm{CI})\end{array}$ & $P$ value \\
\hline 6MWD (m) & $476(434-518)$ & $477(433-521)$ & $1(-33-36)$ & 0.95 & $448(406-490)$ & $543(498-589)$ & $95(58-132)$ & $<0.0001$ & $509(465-553)$ & $546(499-594)$ & $\begin{array}{l}37 \\
(-1-76)\end{array}$ & 0.06 \\
\hline $\begin{array}{r}\mathrm{VO}_{2} \text { peak } \\
(\mathrm{L} / \mathrm{min})\end{array}$ & $1.2(1.0-1.3)$ & $1.3(1.1-1.4)$ & $0.1(0-0.2)$ & 0.08 & $1.1(1.0-1.3)$ & $1.3(1.1-1.4)$ & $0.1(0-0.3)$ & 0.02 & $1.2(1.0-1.3)$ & $1.4(1.2-1.5)$ & $\begin{array}{l}0.2 \\
(0.1-0.3)\end{array}$ & 0.001 \\
\hline Wmax $(\mathrm{W})$ & $81(67-96)$ & $88(73-103)$ & $7(-3-17)$ & 0.1 & $86(72-100)$ & $101(86-116)$ & $15(6-24)$ & 0.002 & $90(75-104)$ & $105(89-120)$ & $15(6-24)$ & 0.002 \\
\hline $\mathrm{QF}(\mathrm{Nm})$ & $86(71-101)$ & $95(79-111)$ & $9(-5-23)$ & 0.2 & $81(66-96)$ & $104(88-120)$ & $23(10-36)$ & 0.0009 & $97(81-112)$ & $105(88-123)$ & $9(-6-23)$ & 0.2 \\
\hline $\begin{array}{l}\text { QLQ-C } 30 \\
\text { Global } \\
\text { (points) }\end{array}$ & $63(51-72)$ & $70(58-81)$ & $8(-1-18)$ & 0.07 & $61(48-71)$ & $68(60-72)$ & $8(-2-17)$ & 0.1 & $62(56-72)$ & $69(60-77)$ & $7(-4-17)$ & 0.2 \\
\hline $\begin{array}{l}\text { QLQ-C } 30 \text { PF } \\
\text { (points) }\end{array}$ & $77(68-85)$ & $78(69-87)$ & $2(-6-9)$ & 0.7 & $70(61-79)$ & $76(67-85)$ & $6(-1-14)$ & 0.09 & $77(68-86)$ & $86(76-95)$ & $8(0-16)$ & 0.04 \\
\hline FACT-F (points) & $13(10--16)$ & $12(8-15)$ & $-1(-5-2)$ & 0.4 & $14(10-17)$ & $10(7-14)$ & $-3(-7-0)$ & 0.08 & $14(10-18)$ & $12(7-16)$ & $-2(-6-2)$ & 0.3 \\
\hline $\begin{array}{l}\text { VAS pain } \\
\text { (points) }\end{array}$ & $2(1-3)$ & $3(1-4)$ & $0(-1-1)$ & 0.8 & $3(2-4)$ & $2(1-3)$ & $-1(-2-1)$ & 0.2 & $3(1-4)$ & $2(1-3)$ & $0(-2-1)$ & 0.6 \\
\hline $\begin{array}{l}\text { VAS dyspnoea } \\
\text { (points) }\end{array}$ & $3(2-4)$ & $2(1-3)$ & $-1(-2--0)$ & 0.08 & $3(2-5)$ & $2(1-3)$ & $-1(-2-1)$ & 0.2 & $4(3-5)$ & $2(1-3)$ & $-2(-3-0)$ & 0.009 \\
\hline
\end{tabular}

M2: after radical treatment, at randomisation; M3: at end of the intervention.

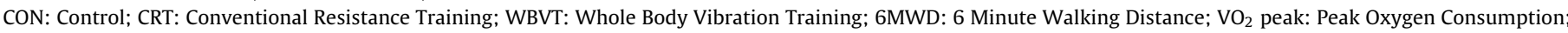

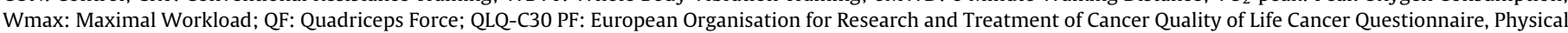
Functioning; FACT-F: Functional Assessment of Cancer Therapy-Scale Fatigue; VAS: Visual Analogue Scale.

a Linear regression: $\triangle \mathrm{M} 3-\mathrm{M} 2$.

Table 4

Comparison of the effect of the different interventions in 70 randomised patients (sample 2).

\begin{tabular}{|c|c|c|c|c|c|c|c|c|}
\hline & \multicolumn{2}{|l|}{ CRT vs. CON } & \multicolumn{2}{|l|}{ WBVT vs. CON } & \multicolumn{2}{|l|}{ CRT vs. WBVT } & \multicolumn{2}{|c|}{ CON vs. CRT and WBVT } \\
\hline & $\begin{array}{l}\Delta \mathrm{M} 3-\mathrm{M} 2 \\
\text { Mean } \\
\text { difference } \\
\text { between } \\
\text { groups }(95 \% \mathrm{CI})\end{array}$ & $P$ value ${ }^{a}$ & $\begin{array}{l}\Delta \mathrm{M} 3-\mathrm{M} 2 \\
\text { Mean } \\
\text { difference } \\
\text { between } \\
\text { groups }(95 \% \mathrm{CI})\end{array}$ & $P$ value & $\begin{array}{l}\Delta \mathrm{M} 3-\mathrm{M} 2 \\
\text { Mean } \\
\text { difference } \\
\text { between } \\
\text { groups }(95 \% \mathrm{CI})\end{array}$ & $P$ value ${ }^{a}$ & $\begin{array}{l}\Delta \mathrm{M} 3-\mathrm{M} 2 \\
\text { Mean } \\
\text { difference } \\
\text { between } \\
\text { groups }(95 \% \mathrm{CI})\end{array}$ & $P$ value \\
\hline 6MWD (m) & $94(43-145)$ & 0.002 & $36(-14-87)$ & 0.480 & $58(5-111)$ & 0.099 & $-66(-21-112)$ & 0.009 \\
\hline $\mathrm{VO}_{2}$ peak $(\mathrm{L} / \mathrm{min})$ & $\begin{array}{l}0.03 \\
(-0.12-0.19)\end{array}$ & 1.000 & $\begin{array}{l}0.08 \\
(-0.08-0.24)\end{array}$ & 0.903 & $0.05(-0.2-0.1)$ & 1.000 & $\begin{array}{l}-0.06 \\
(-0.19-0.08)\end{array}$ & 0.815 \\
\hline $\mathrm{Wmax}(\mathrm{W})$ & $8(-5-21)$ & 0.669 & $8(-5-21)$ & 0.693 & $0(-13-13)$ & 1.000 & $-8(-20-3)$ & 0.334 \\
\hline $\mathrm{QF}(\mathrm{Nm})$ & $14(-4-33)$ & 0.390 & $0(-20-20)$ & 1.000 & $15(-5-34)$ & 0.417 & $-7(-24-10)$ & 0.780 \\
\hline QLQ-C30 Global (points) & $-1(-14-12)$ & 1.000 & $-2(-16-12)$ & 1.000 & $1(-13-15)$ & 1.000 & $1(-10-12)$ & 1.000 \\
\hline QLQ-C30 PF (points) & $5(-6-15)$ & 1.000 & $7(-4-18)$ & 0.693 & $-2(-12-8)$ & 1.000 & $-6(-15-4)$ & 0.472 \\
\hline FACT-F (points) & $-2(-7-3)$ & 1.000 & $-2(-6-5)$ & 1.000 & $-1(-7-5)$ & 1.000 & $2(-3-6)$ & 1.000 \\
\hline VAS pain (points) & $-1(-3-1)$ & 0.873 & $0(-2-1)$ & 1.000 & $-1(-2-1)$ & 1.000 & $1(-1-2)$ & 0.704 \\
\hline VAS dyspnoea (points) & $0(-2-2)$ & 1.000 & $-1(-2-1)$ & 1.000 & $1(-1-3)$ & 1.000 & $0(-1-2)$ & 1.000 \\
\hline
\end{tabular}

M2: after radical treatment, at randomisation; M3: at end of the intervention.

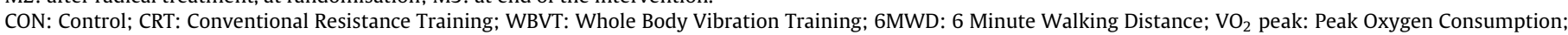

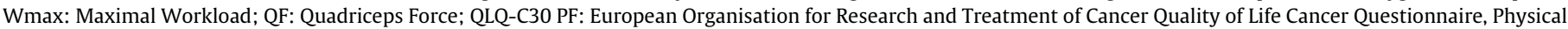
Functioning; FACT-F: Functional Assessment of Cancer Therapy-Scale Fatigue; VAS: Visual Analogue Scale.

a Linear regression: $\triangle \mathrm{M} 3-\mathrm{M} 2$; Bonferonni corrected $P$ values. 


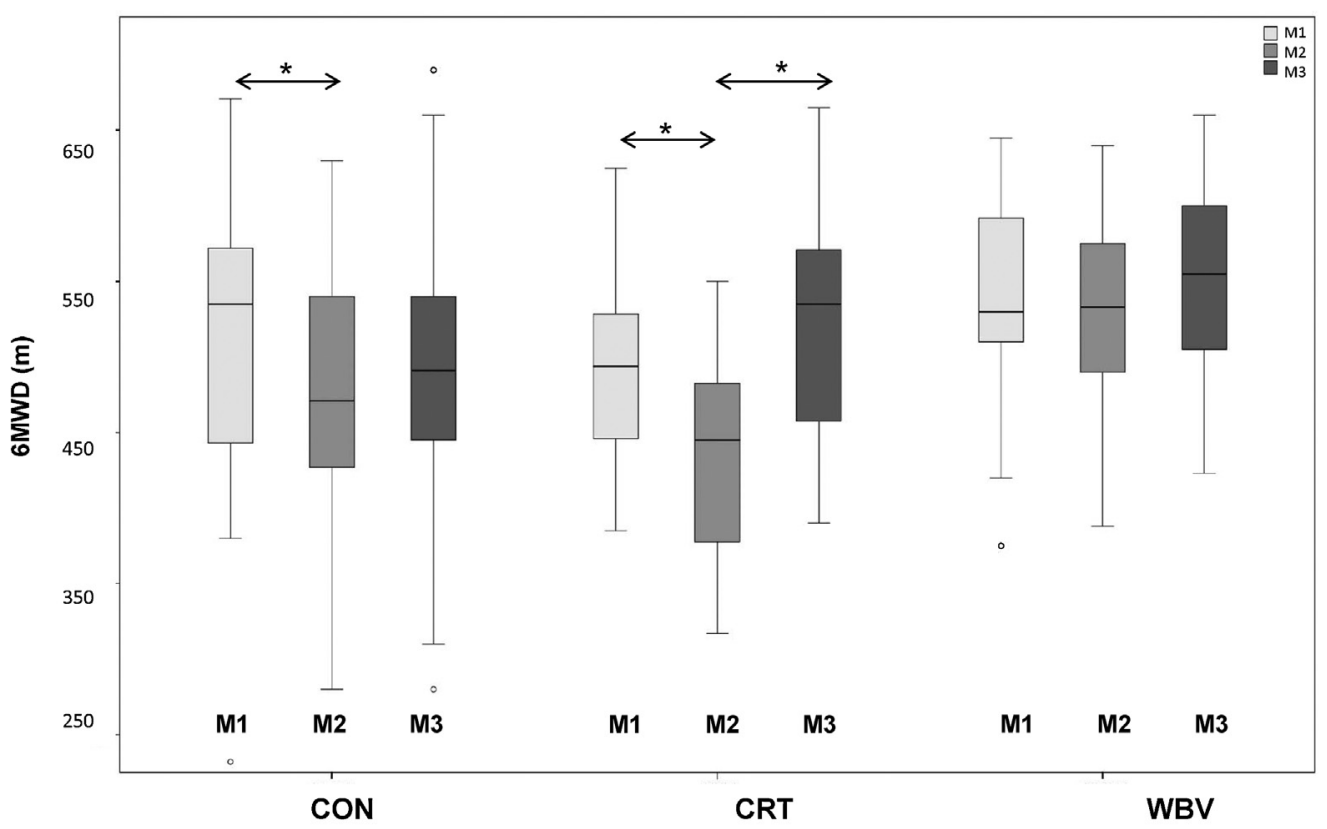

M1: at registration; M2: after radical treatment; M3: after intervention; CON: control group; CRT: conventional resistance training group; WBV: whole body vibration group

*: $<0.05$

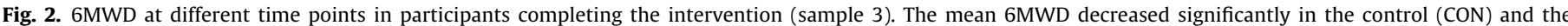

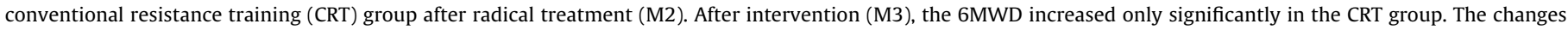
over time observed in the whole body vibration training (WBVT) group were not significant.

Factor

COPD

Rehabilitation (CRT $\div$ WBVT)

Drop of 6MWD (per $50 \mathrm{~m}$ ) by radical treatment (continuous)

\section{Number of patients}

$(\mathbf{N}=58)$

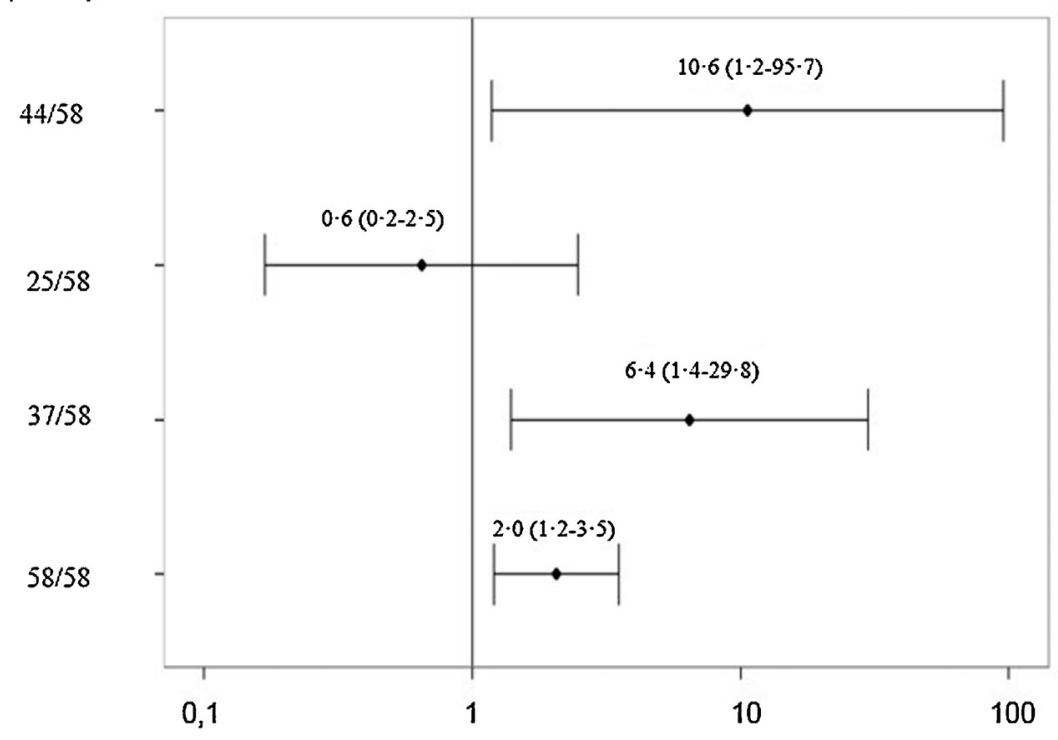

Odds ratio with $95 \% \mathrm{CI}$

MCID: Minimal Clinically Important Difference; 6MWD: 6 Minute Walking Distance; PP: Per Protocol: CoPD: Chronic Obstructive Pulmonary Disease;

CRT: Conventional Resistance Training; WBVT: Whole Body Vibration Training

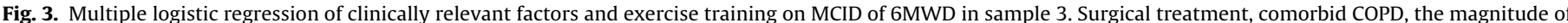

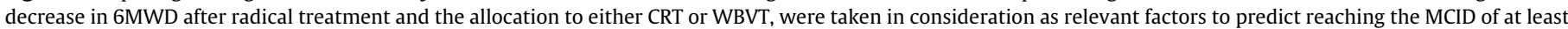

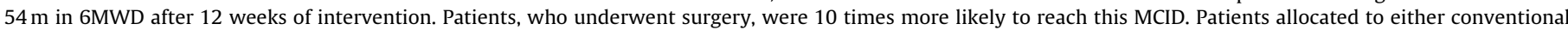

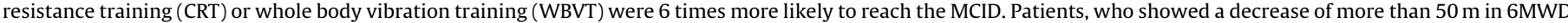
after radical treatment were 2 times more likely to reach this endpoint. 
Forty percent of COPD patients reached the MCID in 6MWD vs. $45 \%$ of patients without COPD ( $\mathrm{p}=0.524$ ). Multiple logistic regression showed that surgical treatment, allocation to either CRT or WBVT and the magnitude of decrease in 6MWD by the radical treatment, were independently associated with reaching the MCID of 6MWD (Fig. 3).

\section{Discussion}

The present study shows that a 12 -week rehabilitation programme in patients with lung cancer significantly improves exercise capacity, muscle strength and QoL, after a previously significant decrease of those variables following treatment with radical intent. The improvement in 6MWD was limited to CRT and was not observed with WBVT. Together with surgery as part of the radical treatment, the magnitude of the decrease in 6MWD by the radical treatment was predictive for reaching the change in 6MWD of $54 \mathrm{~m}$.

Surgery, chemotherapy, and radiation therapy are known to decrease exercise capacity, muscle strength and QoL in lung cancer patients $[15,17,20,27,28,35,36]$. The results of the present study are in line with previously reported data. More importantly, the present study shows that the decrease in 6MWD after radical treatment recovered with CRT and not after usual care. The improvement in 6MWD with CRT largely exceeds the generally accepted threshold of $54 \mathrm{~m}$ reported in COPD patients and ranges between the 40 and $145 \mathrm{~m}$ improvement observed in previous studies in lung cancer patients $[3,14]$. The impact of rehabilitation on 6MWD after thoracotomy for NSCLC was previously investigated in two randomised trials. A home-based training programme, initiated after hospital discharge, yielded no effect [2], whereas an ambulatory programme starting four weeks after surgery resulted in a modest improvement of $35 \mathrm{~m}$ [29]. Possibly, residual post-operative pain may have interfered with training intensity in that trial. In the present study, rehabilitation was not started before the seventh post-operative week, at which time post-operative pain had almost completely disappeared.

The improvement in 6MWD observed with WBVT $(37 \mathrm{~m})$ was neither clinically nor statistically significant. One possible explanation is that the decrease in 6MWD after radical treatment was less pronounced in WBVT than in the other groups, leaving less room for improvement. Another possibility is that WBVT induced an insufficient training effect to improve the 6MWD. Indeed, to increase walking distance, improvement in both muscle strength and muscle power are required, something that is not obtained by performing static exercises on a vibrating platform [21]. Whether longer sets of dynamic training on WBVT are more effective, remain to be investigated.

The 6MWD improved more in patients who underwent a surgical resection than in non-surgically treated patients. Presumably, the decrease in 6MWD in surgically treated patients was due to immediate effects of immobilisation, pain and ventilatory impairment [12], whereas the decrease in functional exercise capacity caused by chemotherapy and radiotherapy may have been long lasting by attenuating normal cardiovascular and/or skeletal muscle adaptation to exercise training [17]. Theoretically, the significantly longer interval between M1 and M2 in non-surgically treated patients could have resulted in a more pronounced deterioration of the general condition. However, the 6MWD at M1 and M2 was not significantly different for both subgroups. That patients with a low 6MWD after radical treatment were more likely to reach the MCID in 6MWD after the intervention, should be attributed to the availability of a larger room for improvement, as has previously been shown in COPD patients [31].
A MCID for maximal exercise capacity in radically treated lung cancer patients has not yet been uniformly defined. Whereas some claim that an increase of $15 \%$ in $\mathrm{VO}_{2}$ max is clinically meaningful, others propose a $10 \%$ increase to be clinically relevant in severe deconditioned and post-operative lung cancer patients [12]. In COPD, a 10-W increase in Wmax is considered a meaningful increase [25]. Although this MCID was reached in the majority of CRT and WBVT-patients, this increase was not statistically significant compared to CON-patients, because the present study was not powered for that outcome.

In rehabilitation, an improvement in $\mathrm{QoL}$ is as important as an increase in physiological outcomes. Conflicting results have been reported on the effect of post-treatment exercise intervention on QoL in patients with lung cancer [14]. In the present study, physical functioning, fatigue and dyspnoea all improved with both training programme, whereas dyspnoea significantly decreased with WBVT. A possible explanation of the beneficial effect of WBVT on dyspnoea is that it promotes aerobic fitness [4].

An important strength of the REINFORCE-study is its multicentre randomised two-tier design with inclusion of consecutive patients, representative for the average cancer population, managed in a department of respiratory medicine. The structured and supervised programme ensured not only the safety of the participants but also assured monitoring of the training intensity. Furthermore, $54 \mathrm{~m}$ is a tougher MCID-threshold than the proposed $35 \mathrm{~m}$ [22]. The included patients were carefully selected in terms of degree of disability, excluding those who did not need rehabilitation, an increasingly important issue in the light of optimal use of health care resources. Lastly, the results of the ITT-analysis were not significantly different from the PP-analysis.

Weaknesses of this study are its high dropout rate, leading to an already low sample size. The dropout rate is comparable to the one observed in our pilot study [3] and reflects the heterogeneity of the impact of the disease and its treatment on fitness, despite thorough staging and a comprehensive radical treatment. The inclusion of non-surgically treated patients may have diluted the observed effect, as surgical patients benefit most from a rehabilitation programme. However, by including such patients, the present trial addresses a group of patients, representative for the larger community of lung cancer patients. Another weakness is the inclusion of three mesothelioma patients whereas the radical treatment intent in these patients is debatable.

Our data question the use of WBVT as a training modality in a rehabilitation programme for radically treated lung cancer patients. Possibly, the present training modality with WBVT did not induce enough fatigue of the quadriceps muscle.

Additionally, a substantial number of patients did not reach the MCID for 6MWD. It remains a challenge to identify patients who do not respond to a rehabilitative intervention. An analysis of the predictive value of biomarkers is ongoing. Future studies should also address the sustainability of the observed improvements. Interestingly, another group is currently investigating the importance of timing, duration and components of an oncological rehabilitation programme in postsurgical lung cancer patients [16].

\section{Conclusion}

This study allows to conclude that a rehabilitation programme should be proposed to all patients with lung cancer treated with radical intent, who exhibit a certain degree of disability related to the malignancy or its treatment. In these patients, rehabilitation improves and restores their functional and maximal exercise capacity, muscle strength and QoL to at least baseline levels. A vibration platform does not fully substitute for conventional multigym equipment. 


\section{Conflicts of interest statement}

We declare no conflict of interest for all the authors and that the grant provider had no influence on the design and outcome of the study, nor on its analysis or content of this manuscript.

\section{Funding}

This work was supported by The Belgian Government Agency of Innovation by Science and Technology for applied Biomedical Research (grant number IWT 070708) and the Clinical Research Fund of Ghent University Hospital, Belgium.

\section{Acknowledgements}

The authors thank the four departments of Respiratory Medicine (Ghent University Hospital, CHU Sart Tilman, Liege, CHU St Pierre, Brussels and AZ Sint Jan, Bruges, Belgium) to participate in the present study. Furthermore the authors thank the members of their rehabilitation team for their dedication and Frank Hulstaert, MD (KCE, Belgium) for a critical reading of the manuscript.

\section{Appendix A. Supplementary data}

Supplementary data associated with this article can be found, in the online version, at http://dx.doi.org/10.1016/j.lungcan.2015.05. 013

\section{References}

[1] Al-Majid S, McCarthy DO. Cancer-induced fatigue and skeletal muscle wasting: the role of exercise. Biol Res Nurs 2001;2:186-97.

[2] Arbane G, Tropman D, Jackson D, Garrod R. Evaluation of an early exercise intervention after thoracotomy for non-small cell lung cancer (NSCLC), effects on quality of life, muscle strength and exercise tolerance: randomised controlled trial. Lung Cancer 2011;71:229-34.

[3] Salhi B, Demedts I, Simpelaere A, Decraene S, Vermeulen K, Surmont V, et al. Endurance and resistance training in radically treated respiratory cancer patients. Rehabil Res Pract 2010:7.

[4] Bogaerts ACG, Delecluse C, Claessens AL, Troosters T, Boonen S, Verschueren SMP. Effects of whole body vibration training on cardiorespiratory fitness and muscle strength in older individuals (a 1-year randomised controlled trial). Age Ageing 2009;38:448-54.

[5] Brooks D, Solway S, Gibbons WJ. ATS statement on six-minute walk test. Am J Respir Crit Care Med 2003;167:1287.

[6] Cella D, Hahn EA, Dineen K. Meaningful change in cancer-specific quality of life scores: differences between improvement and worsening. Qual Life Res 2002;11:207-21.

[7] Charlson ME, Pompei P, Ales KL, Mackenzie CR. A new method of classifying prognostic co-morbidity in longitudinal-studies - development and validation. J Chronic Dis 1987;40:373-83.

[8] Decramer M, Lacquet LM, Fagard R, Rogiers P. Corticosteroids contribute to muscle weakness in chronic airflow obstruction. Am J Respir Crit Care Med 1994;150:11-6.

[9] Edvardsen E, Skjonsberg OH, Holme I, Nordsletten L, Borchsenius F, Anderssen SA. High-intensity training following lung cancer surgery: a randomised controlled trial. Thorax 2015;70:244-50.

[10] Gilliam LAA, St Clair DK. Chemotherapy-induced weakness and fatigue in skeletal muscle: the role of oxidative stress. Antioxid Redox Signal 2011:15:2543-63.

[11] Goldstraw P, Ball D, Jett JR, Le Chevalier T, Lim E, Nicholson AG, et al. Non-smallcell lung cancer. Lancet 2011;378:1727-40.
[12] Gosselink R, Troosters T, Decramer M. Peripheral muscle weakness contributes to exercise limitation in COPD. Am J Respir Crit Care Med 1996;153:976-80.

[13] Gracely RH, Dubner R, McGrath P, Heft H. New methods of pain measurement and their application to pain control. Int Dent J 1978;28:52-65.

[14] Granger CL, McDonald CF, Berney S, Chao C, Denehy L. Exercise intervention to improve exercise capacity and health related quality of life for patients with Non-small cell lung cancer: a systematic review. Lung Cancer 2011;72:139-53.

[15] Hewitt M, Rowland JH, Yancik R. Cancer survivors in the United States: age, health, and disability. J Gerontol Ser A: Biol Sci Med Sci 2003;58:82-91.

[16] Jones LW, Eves ND, Kraus WE, Potti A, Crawford J, Blumenthal JA, et al. The lung cancer exercise training study: a randomized trial of aerobic training, resistance training, or both in postsurgical lung cancer patients: rationale and design. BMC Cancer 2010;10.

[17] Jones LW, Liang YY, Pituskin EN, Battaglini CL, Scott JM, Hornsby WE, et al. Effect of exercise training on peak oxygen consumption in patients with cancer: a meta-analysis (vol. 16, pg 112, 2011). Oncologist 2011;16:112-20.

[18] Jones NL, Makrides L, Hitchcock C, Chypchar T, McCartney N. Normal standards for an incremental progressive cycle ergometer test. Am Rev Respir Dis $1985 ; 131: 700-8$.

[19] MacIntyre N, Crapo RO, Viegi G, Johnson DC, van der Grinten CP, Brusasco V, et al. Standardisation of the single-breath determination of carbon monoxide uptake in the lung. Eur Respir J 2005;26:720-35.

[20] Nezu K, Kushibe K, Tojo T, Takahama M, Kitamura S. Recovery and limitation of exercise capacity after lung resection for lung cancer. Chest 1998;113:1511-6.

[21] Osugi T, Iwamoto J, Yamazaki M, Takakuwa M. Effect of a combination of whole body vibration exercise and squat training on body balance, muscle power, and walking ability in the elderly. Therapeut Clin Risk Manag 2014;10:131-8.

[22] Puhan MA, Mador MJ, Held U, Goldstein R, Guyatt GH, Schunemann HJ. Interpretation of treatment changes in 6-minute walk distance in patients with COPD. Eur Respir J 2008;32:637-43.

[23] Quanjer PH, Tammeling GJ, Cotes JE, Pedersen OF, Peslin R, Yernault JC. Lung volumes and forced ventilatory flows. Report Working Party Standardization of Lung Function Tests, European Community for Steel and Coal. Official statement of the European Respiratory Society. Eur Respir J Suppl 1993;16:5-40.

[24] Redelmeier DA, Bayoumi AM, Goldstein RS, Guyatt GH. Interpreting small differences in functional status: the six minute walk test in chronic lung disease patients. Am J Respir Crit Care Med 1997;155:1278-82.

[25] Ries AL, Make BJ, Lee SM, Krasna MJ, Bartels M, Crouch R, et al. The effects of pulmonary rehabilitation in the national emphysema treatment trial. Chest 2005;128:3799-809.

[26] Rittweger J, Beller G, Felsenberg D. Acute physiological effects of exhaustive whole-body vibration exercise in man. Clin Physiol 2000;20:134-42.

[27] Schag CAC, Ganz PA, Wing DS, Sim MS, Lee JJ. Quality-of-life in adult survivors of lung, colon and prostate-cancer. Qual Life Res 1994;3:127-41.

[28] Schmitz KH, Courneya KS, Matthews C, Demark-Wahnefried W, Galvao DA Pinto BM, et al. American College of Sports Medicine Roundtable on Exercise Guidelines for Cancer Survivors. Med Sci Sports Exerc 2010;42:1409-26.

[29] Stigt JA, Uil SM, van Riesen SJ, Simons FJ, Denekamp M, Shahin GM, et al. A randomized controlled trial of postthoracotomy pulmonary rehabilitation in patients with resectable lung cancer. J Thorac Oncol 2013;8:214-21.

[30] Troosters T, Gosselink R, Decramer M. Six minute walking distance in healthy elderly subjects. Eur Respir J 1999;14:270-4.

[31] Troosters T, Gosselink R, Decramer M. Exercise training in COPD: how to distinguish responders from nonresponders. J Cardiopulm Rehabil 2001;21:10-7

[32] Van Erck A, Vanden Bossche L, Witvrouw E, Van der Kelen V, Wojtowicz I, Adriaenssen J, et al. Effect of whole body vibration on intracompartmental pressure in the lower leg. J Orthop Sci 2009;14:618-22.

[33] van Meerbeeck JP, Fennell DA, De Ruysscher DKM. Small-cell lung cancer. Lancet 2011;378:1741-55.

[34] van Meerbeeck JP, Scherpereel A, Surmont VF, Baas P. Malignant pleura mesothelioma: the standard of care and challenges for future management. Crit Rev Oncol Hematol 2011;78:92-111.

[35] van WE, Hoekstra-Weebers J, Otter R, Postema K, Sanderman R, van der SC. Cancer-related fatigue: predictors and effects of rehabilitation. Oncologist 2006;11:184-96.

[36] Visovsky C. Muscle strength, body composition, and physical activity in women receiving chemotherapy for breast cancer. Integr Cancer Ther 2006:5:183-91.

[37] Yellen SB, Cella DF, Webster K, Blendowski C, Kaplan E. Measuring fatigue and other anemia-related symptoms with the Functional Assessment of Cancer Therapy (FACT) measurement system. J Pain Symptom Manag 1997;13:63-74.

[38] Yuan Y. Multiple imputation using SAS software. J Stat Softw 2011;45:1-25. 